\title{
QUEEN'S
UNIVERSITY
BELFAST
}

\section{2-bit polarisation agile antenna with high port decoupling}

Wolosinski, G., Fusco, V., \& Malyuskin, O. (2016). 2-bit polarisation agile antenna with high port decoupling. Electronics Letters, 52(4), 255-256. https://doi.org/10.1049/el.2015.3701

\author{
Published in: \\ Electronics Letters
}

\section{Document Version:}

Peer reviewed version

Queen's University Belfast - Research Portal:

Link to publication record in Queen's University Belfast Research Portal

\section{Publisher rights}

(C) 2016 Institution of Engineering and Technology Copyright.

This paper is a postprint of a paper submitted to and accepted for publication in Electronics Letters and is subject to Institution of Engineering and Technology Copyright. The copy of record is available at IET Digital Library

\section{General rights}

Copyright for the publications made accessible via the Queen's University Belfast Research Portal is retained by the author(s) and / or other copyright owners and it is a condition of accessing these publications that users recognise and abide by the legal requirements associated with these rights.

Take down policy

The Research Portal is Queen's institutional repository that provides access to Queen's research output. Every effort has been made to ensure that content in the Research Portal does not infringe any person's rights, or applicable UK laws. If you discover content in the Research Portal that you believe breaches copyright or violates any law, please contact openaccess@qub.ac.uk. 


\section{2-bit polarisation agile antenna with high port decoupling}

G. Wolosinski ${ }^{\bowtie}$, V. Fusco and O. Malyuskin

A practical method to achieve both decoupling and six polarisation states by employing the mode-based approach for a four-element antenna is presented. The eigenmode theory as well as a practical implementation scheme are presented. The resulting approach can operate with vertical, horizontal, slant $+45^{\circ}$, slant $-45^{\circ}$, right-hand circular polarisation, or left-hand circular polarisation. A prototype has been manufactured and measured results show good agreement with simulations.

Introduction: In recent years, wireless service providers and base station manufacturers have pushed towards deployment of small cell base stations with stringent performance requirements [1]. Base station antennas with $\pm 45^{\circ}$ slant linear polarisation are routinely used in order to employ polarisation diversity. Generally, the class of antennas used to achieve these polarisation states consist of two crossed radiating elements. Some actual embodiments of this principle are given in $[2,3]$. Recently, there has been interest in a developing modebased strategy in order to decouple closely spaced antennas by generating orthogonal radiation patterns with beamforming [4], the objective being to exploit the antenna eigenmodes to achieve both decoupling and orthogonal radiation patterns in order to increase channel capacity in densely packed antenna scenarios. In this Letter we report what we believe is the first consideration of using the mode-based approach as a means for achieving polarisation agility.

This Letter proposes a simple approach by which to obtain vertical, horizontal, slant $+45^{\circ}$, slant $-45^{\circ}$, right-hand circular polarisation, (RHCP), or left-hand circular polarisation (LHCP) by using a circularly symmetrical antenna attached to a four-port mode decomposition network (MDN). Such polarisation agility and high port-to-port isolation would be very useful in many known and emergent applications, as for example in polarimetric systems [5] and in systems where polarisation diversity can be exploited on a per symbol basis to enhance channel efficiency [6].

Theory and implementation: In the following approach any fourelement antenna with circular symmetry can be used as many base station antennas fulfil this requirement $[2,3]$. Here, we use an antenna consisting of four capacitively fed monopoles excited through coaxial ports arranged symmetrically as shown in Fig. 1.

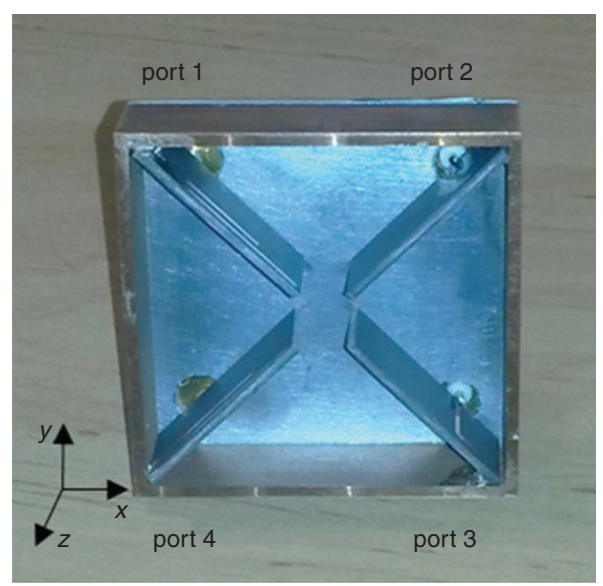

Fig. 1 Photograph of prototype with port numbers

The scattering matrix of such an arrangement takes the form

$$
\boldsymbol{S}_{A}=\left(\begin{array}{cccc}
A & B & C & B \\
B & A & B & C \\
C & B & A & B \\
B & C & B & A
\end{array}\right)
$$

where $A=S_{11}=S_{22}=S_{33}=S_{44}$ are the reflection coefficients of each element, $B=S_{12}=S_{14}=S_{21}=S_{23}=S_{32}=S_{34}=S_{41}=S_{43}$ are the coupling coefficients between adjacent elements, and $C=S_{13}=S_{31}=S_{24}=S_{42}$ are the coupling coefficients between opposite elements. The mode-based approach leads to a network that can act to suppress the mutual coupling of $\boldsymbol{S}_{A}$ [4] by applying the following condition

$$
\boldsymbol{S}_{D}=\boldsymbol{P}^{-1} \boldsymbol{S}_{A} \boldsymbol{P}
$$
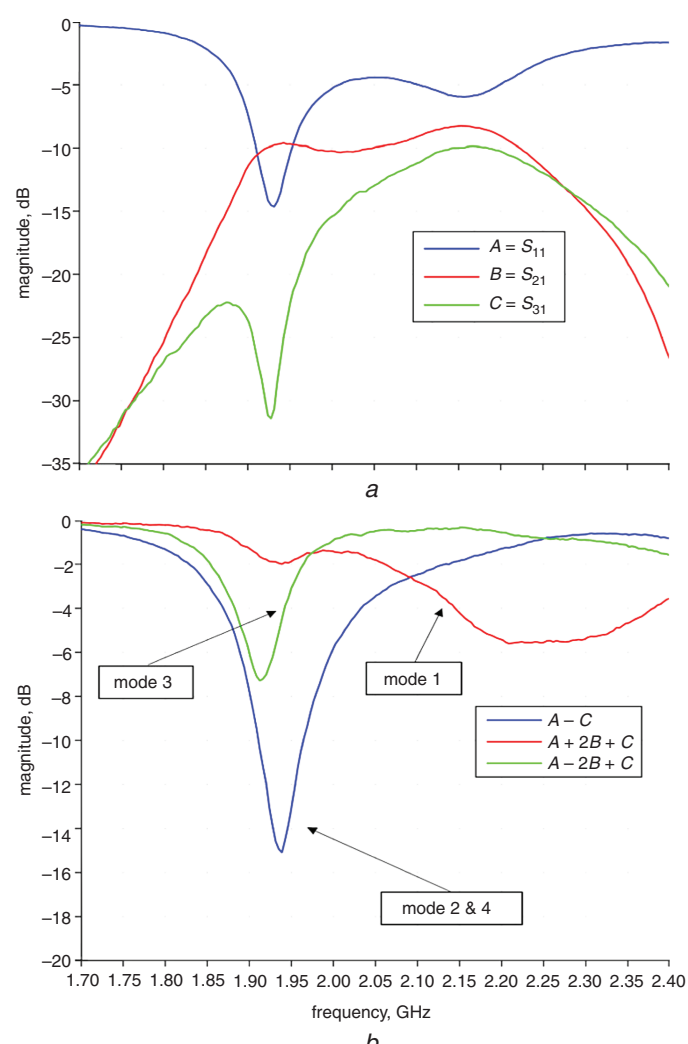

Fig. 2 S-parameters

a Measurement of antenna in Fig.

$b$ Computed eigenvalues obtained after diagonalisation

where $\boldsymbol{S}_{D}$ is a diagonal matrix containing all eigenvalues of $\boldsymbol{S}_{A}, \boldsymbol{P}$ is an orthonormal matrix consisting of the corresponding eigenvectors, and $\boldsymbol{P}^{-1}$ is the matrix inverse of $\boldsymbol{P}$. Here, $\boldsymbol{P}$ represents the MDN and takes the form in (3).

$$
\begin{gathered}
\boldsymbol{P}=\frac{1}{2}\left(\begin{array}{cccc}
+1 & +1 & +1 & +1 \\
+1 & +1 & -1 & -1 \\
+1 & -1 & +1 & -1 \\
+1 & -1 & -1 & +1
\end{array}\right) \\
\boldsymbol{P}^{\mathrm{T}}=\boldsymbol{P}^{-1}
\end{gathered}
$$

The diagonalisation condition in (2) is subject to the physical realisability condition in (4), thus the MDN can be implemented using four cross connected $180^{\circ}$ hybrid couplers (see Fig. 4). The cascade of the antenna coaxial ports with the MDN results in a diagonal matrix $S_{D}$ which has entries as follows

$$
\boldsymbol{S}_{D}=(A+2 B+C, A-C, A-2 B+C, A-C)
$$

Although the theoretical mutual coupling is suppressed, a real $180^{\circ}$ hybrid coupler [7] has been considered to simulate a MDN and give a real estimate of the decoupling. The measured $S$-parameters of the antenna (see Fig. 2a) in cascade with the above-mentioned MDN provide a mutual coupling better than $-30 \mathrm{~dB}$ within a range of $400 \mathrm{MHz}$ around the operating frequency of $1.93 \mathrm{GHz}$. The input ports of the MDN have a new reflection coefficient according to (5), therefore port matching is typically required. The eigenvalues for each mode are reported in Fig. $2 b$.

A schematic representation of the excited currents on the antenna elements for each of the four modes is reported in Fig. 3. Modes 1 and 3 provide a null in the radiation pattern at boresight and yield mismatch according to Fig. $2 b$. On the other hand, modes 2 and 4 provide constructive interference in the desired direction while preserving also 
the matching condition at the operating frequency. For these reasons, in the rest of the discussion we focus on using modes 2 and 4

Fig. 3 shows how modes 2 and 4 can be used both separately and in superposition in order to generate the primary four linear polarisation states. In addition, two circular polarisation states can also be generated. Each of these can be obtained by individually stimulating the relevant input port of the MDN, Fig. 4 according to Table 1, wherein all of the excitation states can be obtained using only two input ports of the MDN and a two-bit phase shifter connected to the mode 4 port. mode 1

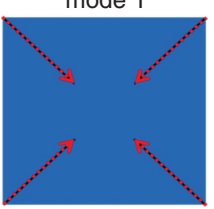

mode 2

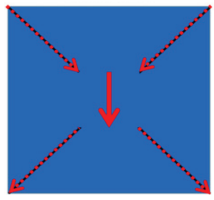

mode 3

mode 4

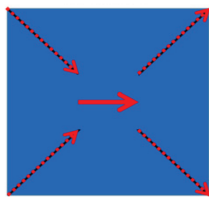

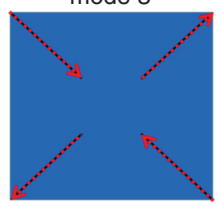

mode $2+4$

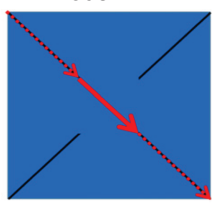

mode $2+4\left(180^{\circ}\right)$

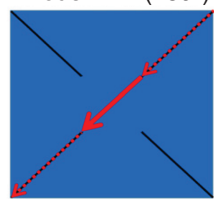

Fig. 3 Available linear polarisation states by using mode 2 and 4 port. Arrows show principal current direction for each antenna element and resulting polarisation. Port numbering as in Fig. 1

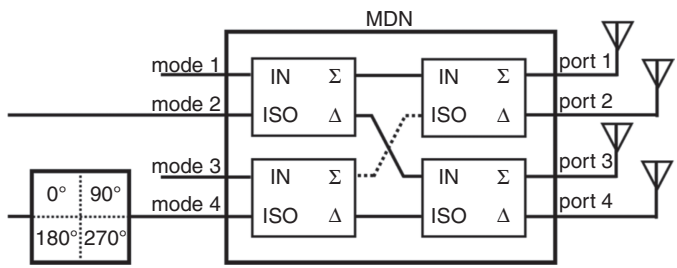

Fig. 4 Principle scheme for generating polarisation states in Table 1. From left to right: 2-bit phase shifter, MDN (hybrid implementation), and fourelement antenna

Table 1: Required excitation for each polarisation

\begin{tabular}{|c|c|}
\hline Polarisation & Excitation \\
\hline Vertical & Mode 2 \\
\hline Horizontal & Mode 4 \\
\hline Slant $-45^{\circ}$ & Mode $2+4$ \\
\hline Slant $+45^{\circ}$ & Mode $2+4\left(-180^{\circ}\right)$ \\
\hline RHCP & Mode $2+4\left(-90^{\circ}\right)$ \\
\hline LHCP & Mode $2+4\left(-270^{\circ}\right)$ \\
\hline
\end{tabular}

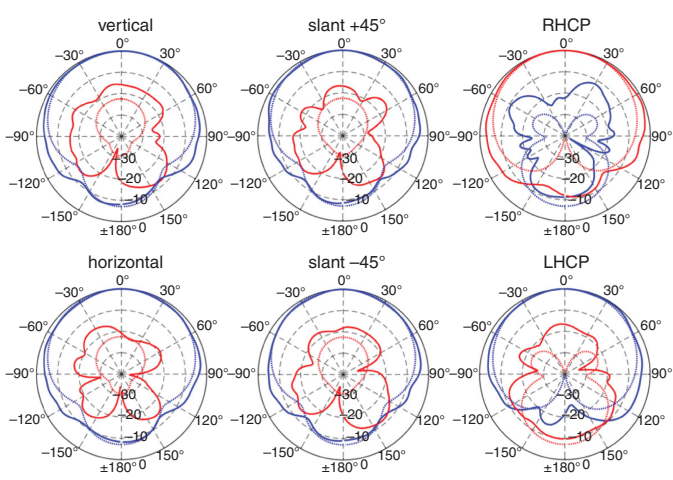

Fig. 5 Radiation patterns at $1.93 \mathrm{GHz}$. Solid and dotted lines are measured and simulated data, respectively. Co-polar (blue lines) and cross-polar (red lines) for vertical, horizontal, slant $+45^{\circ}$, and slant $-45^{\circ}$. Right-handed (red lines) and left-handed (blue lines) circular polarisation for RHCP and LHCP
Validation: In this Section we validate the proposed theory through measurements. The antenna was measured in an anechoic chamber scanning along $\varphi=0^{\circ},+45^{\circ},-45^{\circ}$, and $+90^{\circ}$. The frequency of the measurement was selected to be $1.93 \mathrm{GHz}$. The antenna was fixed on a plastic support and no additional ground plane was employed. Each port was measured individually while the others were terminated in a $50 \Omega$ load. Thereafter, the data sets were combined according to the MDN properties in order to synthesize the radiation patterns for each polarisation.

In Fig. 5 shows the simulated and experimentally obtained radiation patterns for each polarisation normalised at boresight to $0 \mathrm{~dB}$. The radiation patterns for the linear polarisation are plotted along the E-plane while the circular ones on $\varphi=90^{\circ}$. The measured results are in a good agreement with the simulations although a higher level of crosspolarisation is observed which can be attributed to the manufacturing defects of the antenna and a partial misalignment of the antenna phase centre during the measurement stage. In addition, a slight ripple can be noted which is expected to be from the reflections of the holder structure.

Conclusion: In this Letter the mode-based approach has been employed in a four-element circularly symmetrical antenna in order to obtain decoupling and polarisation agility. The mutual coupling has been estimated to be below $-30 \mathrm{~dB}$ within a range of $400 \mathrm{MHz}$ around the functioning frequency while any one of six possible polarisation states can be selected by the combination of only two ports of the MDN with the aid of a two-bit phase shifter. Simulated and measured results have proven the feasibility that vertical, horizontal, slant $+45^{\circ}$, slant $-45^{\circ}$, RHCP, and LHCP polarisation can be readily achieved. The resulting approach would be useful in a polarimetric system or in an advanced polarisation encoded communication system.

Acknowledgments: This work was supported by the Marie Curie European Industrial Doctorate (EID) programme ARTISAN (grant no. 316426). The authors thank V. Basavarajappa for the design of the antenna structure.

(C) The Institution of Engineering and Technology 2015

Submitted: 25 October 2015

doi: 10.1049/el.2015.3701

One or more of the Figures in this Letter are available in colour online. G. Wolosinski, V. Fusco and O. Malyuskin (ECIT Institute, Queens University Belfast, Queens Road, Belfast, BT9 3DT, United Kingdom)

$凶$ E-mail: g.wolosinski@qub.ac.uk

\section{References}

1 Bottai, C., Cicconetti, C., Morelli, A., Rosellini, M., and Vitale, C.: 'Energy-efficient user association in extremely dense small cell networks'. EuCNC, Manchester, UK, June 2014, pp. 1-5

2 Asrokin, B., Bin Abas, A., Bin Basri, R.H., and Bin Jamlus, N.: 'Design of x-polarized GSM 900 base station antenna with field test measurement'. ICCEA 2010, Bali Island, Indonesia, April 2010, Vol. 2, pp. $94-98$

3 Cui, Y.H., Li, R.L., and Fu, H.Z.: 'A broadband dual-polarized planar antenna for 2G/3G/LTE base stations', IEEE Trans. Antennas Propag., 2014, 62, (9), pp. 4836-4840

4 Yeung, L.K., and Wang, Y.E.: 'Mode-based beamforming arrays for miniaturized platforms', IEEE Trans. Microw. Theory Tech., 2009, 57, (1), pp. $45-52$

5 Sarabandi, K., Ulaby, F., and Tassoudji, M.: 'Calibration of polarimetric radar systems with good polarization isolation', IEEE Trans. Geosci. Remote Sens., 1990, 28, (1), pp. 70-75

6 Abidin, Z., Xiao, P., Amin, M., and Fusco, V.: 'Circular polarization modulation for digital communication systems'. 8th Int. Symp. Communication Systems, Networks, and Digital Signal Processing (CSNDSP), Poznan, Poland, July 2012, pp. 1-6

7 Anaren n.d., www.anaren.com/sites/default/files/3A0200.pdf 